just as it is during REM sleep. Additionally, cataplexy-enhancing drugs reduce the firing activities of these two neuronal groups.

The investigators now show that, unlike these other two types of REM-off nuclei, the histamine-containing neurons in the TMN have firing rates during cataplexy that resemble those during quiet waking; moreover, administration of the cataplexyenhancing drugs does not alter the activity of these neurons ${ }^{1}$. The authors go on to show that release of GABA (an inhibitory neurotransmitter) in the TMN is substantially lower during cataplexy than during REM, consistent with increased activity. Thus, during the pathological state of cataplexy, the histaminergic TMN neurons have activities that correlate with alertness, whereas the activity of the other two cell groups correlates with loss of muscle tone.

Siegel and colleagues postulate that the activity of at least one of these two cells groups, the noradrenergic REM-off cells of the LC, determines muscle tone. These neurons express Hcrtr1, and their activity is driven by elevated hypocretin release during wakefulness as well by as excitatory input from other sources. Although emotion can inhibit the activity of LC neurons in both normal individuals and narcoleptics
(Fig. 1), lack of Hcrt excitation in human narcoleptics results in loss of muscle tone. Consistent with this scheme, stress, which activates noradrenergic input and sympathetic tone, inhibits cataplexy.

Although LC activation was once thought to correlate with cortical arousal, there is now ample evidence, including lack of activity during cataplexy, that LC activation correlates with muscle tone and motor control. Nevertheless, there is little doubt that, under normal conditions, LC neurons contribute substantially to vigilance in addition to controlling muscle tone.

The findings that REM-off histaminergic cells are active during cataplexy, and might be driven by Hcrt-independent, excitatory inputs, fit with the idea that the firing of histaminergic neurons correlates with environmental awareness. The findings also explain why Hcrtr2-deficient mice, which lack the excitatory input to histamine cells, are constantly sleepy.

This scheme, however, still presents a puzzle: If Hcrtr2-expressing cells mediate alertness, and alertness does not dim during cataplexy, why do Hcrtr2-deficient narcoleptic dogs exhibit cataplexy? Siegel and colleagues invoke an indirect effect, speculating that the absence of Hcrtr2 in the hypothalamus somehow reduces the excitation of LC neurons by Hcrt, and these neurons cannot compensate when they receive emotion-elicited inhibition. However, when the histamine cells of the TMN remain active, their activity is sufficient to maintain consciousness. In normal individuals, the excitation of the TMN by Hcrt maintains vigilance during waking, and antihistamines reduce alertness by defeating this system.

The difference between the sleeping and waking states is complex, but studies on animal models of narcolepsy have been instructive. The aroused state requires both mental alertness and muscle tone, and these are driven by the sum of the activities of the various key brain regions. Both the Hcrtr1 and Hcrtr2 branches of the hypocretin system are crucial for coordinating these activities, suggesting that small-molecule agonists of these two receptors might have therapeutic value in narcolepsy.

1. John, J. et al. Neuron 42, 619-634 (2004).

2. Sutcliffe, J.G. \& de Lecea, L. Nat. Rev. Neurosci. 3, 339-349 (2002)

3. Lin, L. et al. Cel/ 98, 365-376 (1999).

4. Chemelli, R.M. et al. Cell 98, 437-451 (1999).

5. Nishino, S. et al. Lancet 355, 39-40 (2000).

6. Peyron, C. et al. Nature Med. 6, 991-997 (2000).

7. Thannickal, T.C. et al. Neuron 27, 469-474 (2000).

8. Willie, J.T. et al. Neuron 38, 715-730 (2003).

\title{
Sleep smarts
}

The idea that memory consolidation is one function of sleep is quite old. So far, the evidence in favor of this notion is correlative: performance of a task that is learned before a good night's sleep improves the next morning. Reporting in Nature, Reto Huber et al. now show that areas of the brain that are relevant for learning experience local synaptic changes during sleep, and propose that these changes might benefit performance.

The researchers trained humans in a motor task known to activate mainly one region of the brain - the parietal lobe. They then measured electrical activity during sleep using 256 electrodes distributed over the skull, as shown here. Huber et al. were particularly interested in the slow wave activity (SWA) that appears during sleep. The longer we stay awake, the higher the pressure for sleep and the higher the proportion of SWA during the sleeping period. But the relationship between SWA and memory consolidation had not been explored, and the neuroanatomical specificity of the task provided these researchers with the ideal tool to address this question.

Huber et al. found that SWA increased over the parietal lobe (larger dots in the figure) in trained as compared to untrained subjects. What's more, the magnitude of this increase correlated with improved performance the next morning. These results indicate that the relationship between SWA and learning is tighter than previously believed. Although the evidence for a role of sleep in memory consolidation remains correlative, as far as correlations go, it doesn't get much better than this.

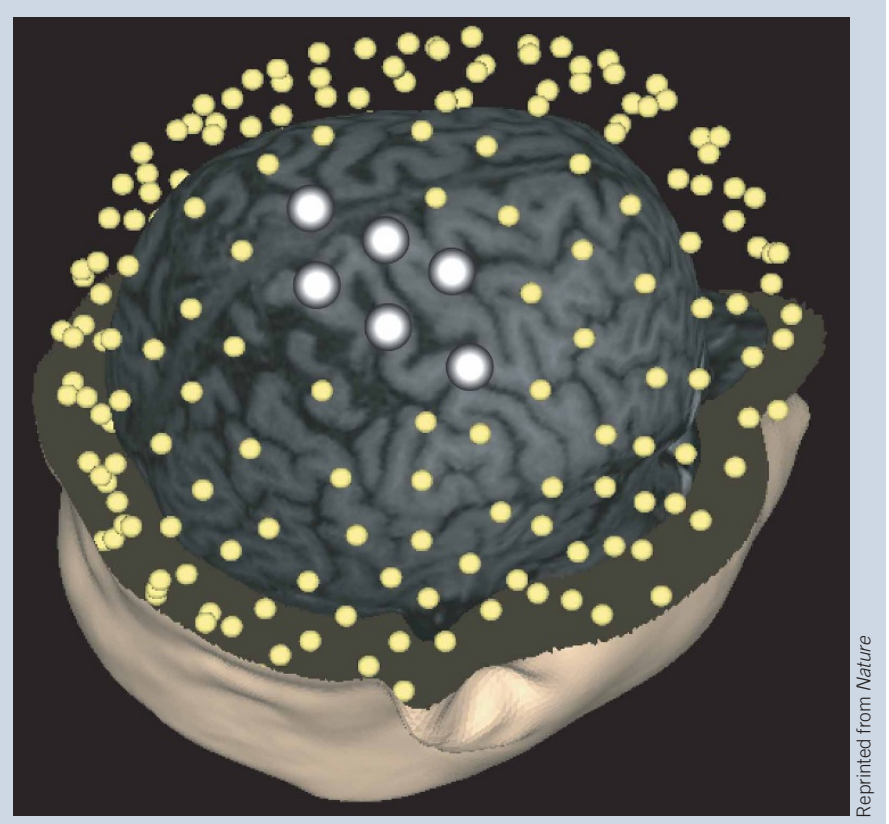

Juan Carlos López 